\title{
Highly stereoselective syntheses of arsenic and phosphorus macrocycles and cages
}

\author{
S. Bruce Wild
}

Research School of Chemistry, Australian National University, Canberra, Australian Capital Territory, Australia 2601

\begin{abstract}
The dimerization of certain 2-mercaptoethyl and 2-aminoethyl substituted tertiary arsines, chiral at arsenic, under appropriate condition produces high yields of 14-membered trans-As $\mathrm{S}_{2}$ or trans- $\mathrm{As}_{2} \mathrm{~N}_{2}$ macrocycles, with high stereoselectivities. The optical isomers of the macrocycles have also been isolated. Highly stereoselective syntheses of coordinated secondary and tertiary arsines and phosphines have also been performed. The first resolution of secondary phosphine, chiral at phosphorus, has been achieved by the method of metal complexation and a tricyclic tetra(tertiary phosphine) cage has been synthesised from 1,2-phenylene(bisphosphine) on copper(I) in the presence of base. Moreover, it has been shown that that the mould Scopulariopsis brevicaulis reductively methylates ethyl-impropylarsinic acid with production of ethylmethyl-n-propylarsine of ca $80 \%$ optical purity.
\end{abstract}

\section{INTRODUCTION}

Asymmetric synthesis, once considered a rather exotic speciality in chemistry, is now a primary focus of activity for academic and industrial chemists (ref, 1). The asymmetric synthesis of one enantiomer or of one diastereomer of a substance is a very attractive proposition, because unlike a chemical resolution or separation the yield of the stereoisomer can be $100 \%$. Although dominated by organic chemists employing natural products and their derivatives as chiral auxiliaries, the field is wide open to coordination chemists, who, with their superior knowledge of ligands and the chelate effect, have before them unparalleled opportunities for the development of stereoselective reactions. In this lecture I will show that highly stereoselective syntheses of optically active and diastereomerically homogeneous tertiary arsines ( $E_{i n y}>40 \mathrm{kcal} \mathrm{mol}^{-1}$ ) and tertiary phosphines $\left(E_{\text {inv }} 30-35 \mathrm{kcal} \mathrm{mol}^{-1}\right.$ ) (ref. 2) are now possible, and that it is a realistic proposition for chemists to design and manufacture particular stereoisomers of tertiary arsines and phosphines, chiral at arsenic or phosphorus, for use in asymmetric synthesis. In this paper, emphasis will be placed on those reactions of chiral trivalent arsenic and phosphorus compounds that proceed with complete stereoselectivity (Note a).

\section{MACROCYCLIC TERTIARY ARSINES}

Given the wealth of chemistry, both organic (ref. 3) and inorganic (ref. 4) based upon the macrocyclic chelate effect on the one hand and the coordination chemistry of tertiary arsines on the other, it is surprising that few fully characterised macrocyclic tertiary arsines are known (ref. 5). In recent work, we showed that highly stereoselective syntheses of 14-membered macrocycles containing two chiral tertiary arsine groups occur in

Note a: The term "complete stereoselectivity", or an equivalent expression, will henceforth mean that a synthesis has yielded a diastereomerically homogeneous product, as determined by high resolution NMR spectroscopy, or an enantiomerically homogeneous product, as determined by an appropriate method. 
couplings of appropriate mono-tertiary arsines. For example, ( \pm ) -1 (L) readily forms the complex $\left[\mathrm{Pd}(\mathrm{L}-\mathrm{H})_{2}\right]$, which, although it exists in solution as an equilibrium mixture of four diastereomers, yields, upon treatment with boron tribromide, diastereomerically pure $\left(R^{*}, R^{*}\right)-2$ (Note b) after removal of the metal (ref. 6). Although $R^{*}, S^{*}$ diastereomers of $\left[\mathrm{Pd}(\mathrm{L}-\mathrm{H})_{2}\right]$ are present in the reaction mixture, and there are six possible
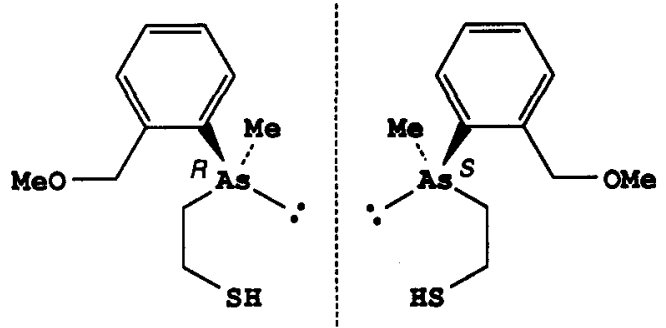

$( \pm)-1$

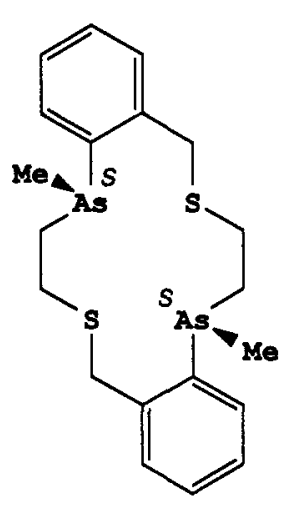

$(R *, R *)-( \pm)-2$

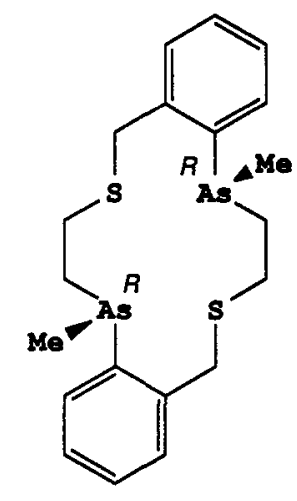

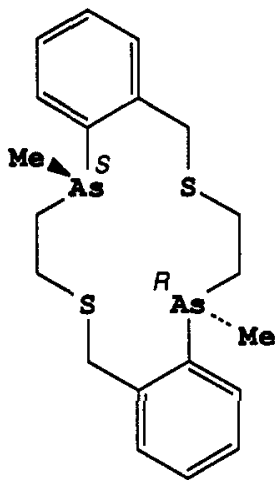

$\left(R^{*}, S^{*}\right)-2$

diastereomers of $[\mathrm{Pd}(2)]^{2+}$ (the sulfurs are also chiral), one diastereomer of the macrocyclic complex is produced to the exclusion all others. Interestingly, pure $\left(R^{*}, R^{*}\right)-$ 2 , when dissolved in chloroform to which a trace of aqueous hydrochloric acid has been added, rearranges with epimerization at arsenic to give an equilibrium mixture of $\left(R^{*}, R^{*}\right)$ 2 , and $\left(R^{*}, s^{*}\right)-2$. Under suitable conditions diastereomerically pure $\left(R^{*}, s^{*}\right)-2$ crystallizes from the acidic solution in quantitative yield in a typical second-order asymmetric transformation. On the other hand, if $\left(R^{*}, S^{*}\right)-2$ is converted into $\left[\operatorname{Pd}\left(R^{*}, S^{*}\right)-\right.$ 2] $\left(\mathrm{ClO}_{4}\right)_{2}$, and this complex is heated at $150{ }^{\circ} \mathrm{C}$ in dimethylsulfoxide for a few minutes, the coordinated arsines epimerise with quantitative formation of diastereomerically pure [Pd $\left.\left(R^{*}, R^{*}\right)-2\right]\left(\mathrm{ClO}_{4}\right)_{2}$. Thus, by appropriate choice of conditions either diastereomer of 2 can be interconverted quantitatively with complete stereoselectivity.

Note b: The stereochemical nomenclature adopted here for diastereomers is consistent with Chemical Abstracts Service Index practice; $R^{*}$ and $S^{*}$ refer to the relative absolute configurations of the chiral centres. Enantiomers have simplified descriptors. 
If ( \pm$)-1$ is first resolved (and this can be achieved by the fractional crystallization of optically active palladium(II) diastereomers of deprotonated ( \pm ) -1, followed by displacem[5 ents of the optically pure arsine enantiomers from the diastereomers) and then converted into optically active $\left[\mathrm{Pd}(\mathrm{L}-\mathrm{H})_{2}\right]$, cyclisation yields optically pure $(R, R)-$, or (S.S)-2, the first examples of optically active macrocyclic tertiary arsines.<smiles>CN1CCNC1c1ccccc1[As](C)CCN</smiles>

(士) -3

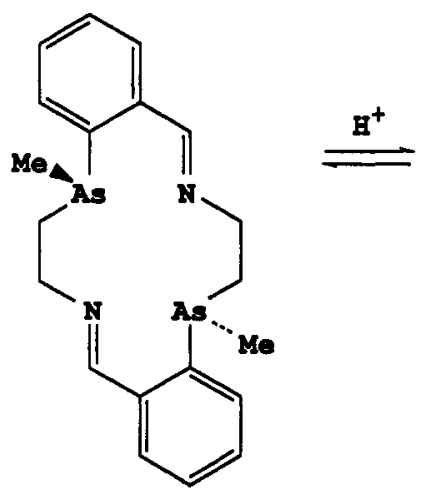

$\left(R^{\star}, S^{\star}\right)-4$<smiles>COC(=O)c1ccccc1C=NCCO</smiles>

(士) -5

We have also isolated pure diastereomers and enantiomers of trans- $\mathrm{As}_{2} \mathrm{~N}_{2}$ macrocycles (ref. $7)$. In a dramatic demonstration of stereospecificity, $( \pm)-3$, when heated at $80^{\circ} \mathrm{C}$ in vacuo, yields diastereomerically pure $\left(R^{*}, s^{*}\right)-4$ in quantitative yield. The difmine rearranges quantitatively into 7-membered $( \pm)-5$ in chloroform; upon removal of solvent, $\left(R^{*}, S^{*}\right)-4$ is recovered. Thus, $\left(R^{*}, R^{*}\right)-4$ cannot be isolated and the synthesis of $\left(R^{*}, S^{*}\right)-4$ from $( \pm)-3$ is totally stereospecific. The individual enantiomers of $\left(R^{*}, R^{*}\right)-4, v i z$. $(R, R)$ - and $(S, S)-4$, however, can be isolated by asymmetric synthesis and each one is optically pure. Under acidic conditions $\left(R^{*}, S^{*}\right)-4$ reacts with $R-6$ to produce, after a work-up involving treatment of the reaction mixture with ammonium hexafluorophosphate, a diastereomerically pure palladium complex containing $(R, R)-4$. 0ptically pure $(R, R)-4$ can be liberated from the complex. Reductions of $\left(R^{*}, s^{*}\right)-$ and $(R, R)-4$ with lithium aluminium hydride yield the corresponding diamines. Since $\left(R^{*}, R^{*}\right)-4$ is not avallable, the racemic diamine was obtained by heating $\left[\mathrm{Pd}\left(R^{*}, S^{*}\right)-4\right]\left(\mathrm{ClO}_{4}\right)_{2}$ in dimethylsulfoxide for a few minutes at $150{ }^{\circ} \mathrm{C}$, and then liberating from the metal the $\left(R^{*}, R^{*}\right)-4$ that had formed quantitatively by asymmetric transformation [see $\left(R^{*}, S^{*}\right)-2 \rightarrow\left(R^{*}, R^{*}\right)-2$ ]

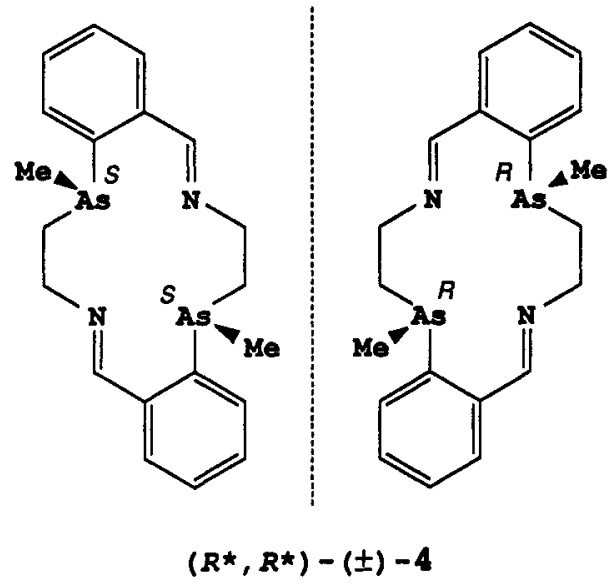

In recent work, we have explored the possibilities of resolving and stereoselectively alkylating secondary arsine complexes (ref. 8). Secondary arsine ( \pm )-AsHMePh reacts with 7 in boiling methanol to give, in a second-order asymetric transformation, a $90 \%$ yield of crystalline $R-8$, diastereomerically pure, the first example of a complex containing a resolved secondary arsine. Deprotonation of the secondary arsine in this complex with 


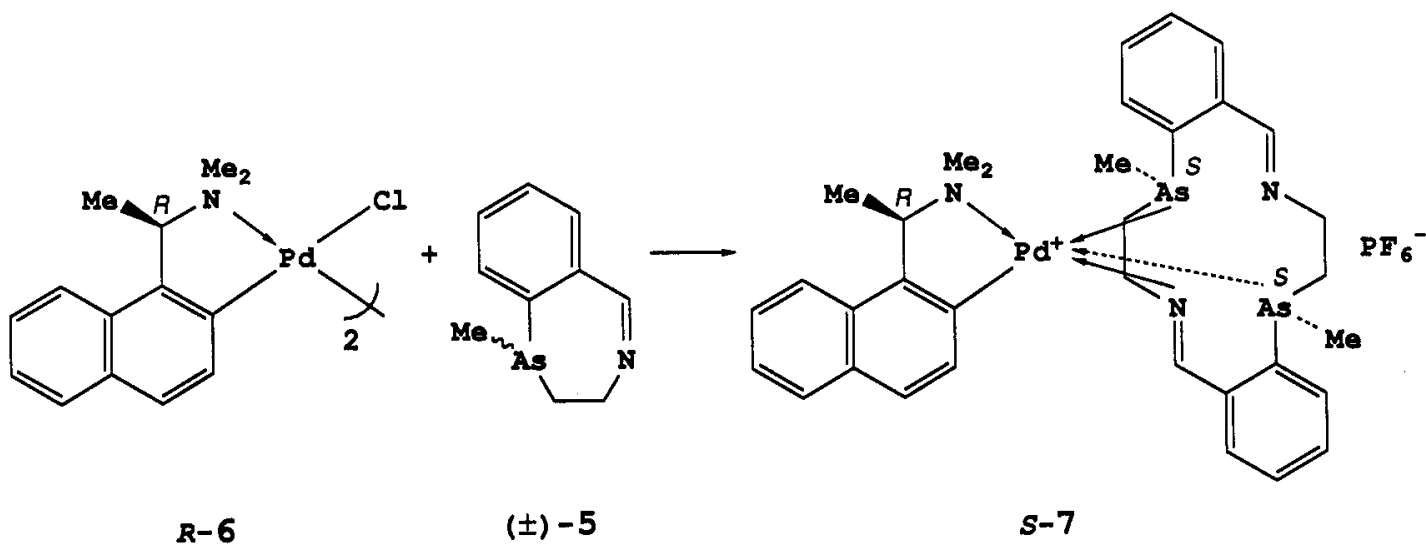

$\mathrm{KOBu}-1$ at $-65^{\circ} \mathrm{C}$ followed by treatment of the intermediate tertiary arsenido-iron intermediate with ethyl iodide at the same temperature, yields diastereomerically pure (5)-9. Thus, it is possible to synthesise a tertiary arsine from a free secondary arsine with total stereoselectivity with use of the auxiliary 7. In another development, we have found that 7 reacts with $( \pm)-A s C l M e P h$ to give an equilibrium mixture of the expected chloroarsine complexes. Treatment of tetrahydrofuran solutions of this mixture of epimers with Grignard reagents gives the diastereomerically pure tertiary arsine complexes in > $90 \%$ chemical yield. Grignard reactions on diastereomerically homogeneous chlorophosphine complexes, chiral at phosphorus, also proceed with complete ( $>99 \%$ ) stereoselectivity, apparently with inversion at phosphorus. In another development, we have isolated a platinum(II) complex containing a resolved secondary phosphine that can be liberated with retention of configuration at phosphorus; this is the first resolution of a chiral secondary phosphine. These results auger well for future stereoselective syntheses of pure diastereomers and enantiomers of poly(tertiary arsines and phosphines) on metal ions.

\section{TERTIARY PHOSPHINE MACROCYCLES AND CAGES}

We have been concerned with the synthesis of chelating poly(tertiary phosphine) macrocycles and cages for some time. Related work in this area has emanated from Kyba's laboratories (ref. 9). One strategy for the synthesis of Irans- $\mathrm{P}_{2} \mathrm{~S}_{2}$ macrocycles involves the metal template dimerization of appropriately substituted $( \pm)-2$-mercaptoethylphosphines along the lines that proved successful for the synthesis of the diastereomers and

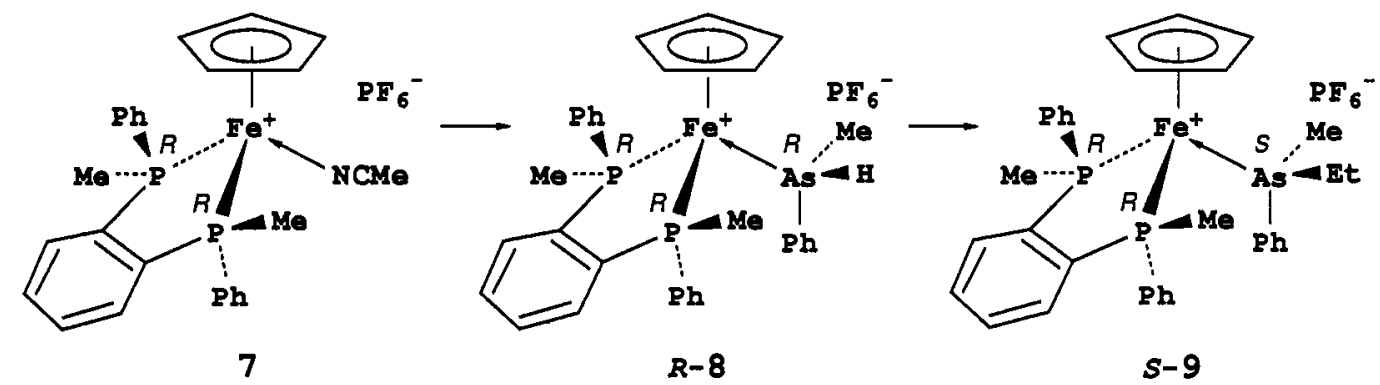

enantiomers of 2 . Another approach has been to stereoselectively alkylate appropriate primary and secondary phosphines in order to build-up rings at phosphorus. To date, our work on the first approach has resulted in the resolution of the model compound $( \pm)$ PhMePCH $\mathrm{CH}_{2} \mathrm{SH}$ with use of $R-6$ as resolving agent and the corresponding 2-methoxymethyl compound, as a racemate, has been cyclized on platinum(II) (as for 2) giving low yields of the $\left(R^{*}, R^{*}\right)-( \pm)$ and $\left(R^{*}, S^{*}\right)$ forms of trans- $\mathrm{P}_{2} \mathrm{~S}_{2}$ macrocycles. 


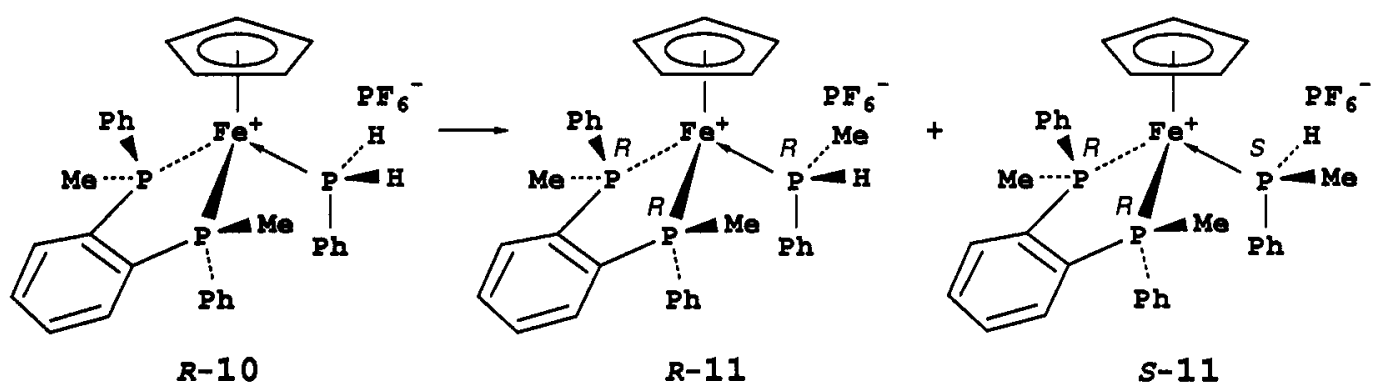

The second approach, which involves ring build-up by stereoselective alkylation of coordinated primary and secondary phosphines, seems particularly promising. Thus, reaction of $R-10$ with iodomethane in the presence of triethylamine at $20^{\circ} \mathrm{C}$ produces a separable 4:1 mixture of $R$ - and $S-11$ (ref. 10). Deprotonation of $R-11$ with KOBu- $t$ at $-90{ }^{\circ} \mathrm{C}$, followed by treatment of the intermediate tertiary phosphido-iron complex with iodoethane at the same temperature, produces $\$-12$ with complete stereoselectivity (refs. 11 and 12). Resolved secondary phosphine complexes can be converted therefore into resolved tertiary phosphine complexes with total stereochemical control at low temperatures. At elevated temperatures alkylations proceed under thermodynamic control due to the relatively low barrier to inversion of the tertiary phosphido-metal- $P$ stereocentres in the intermediates.

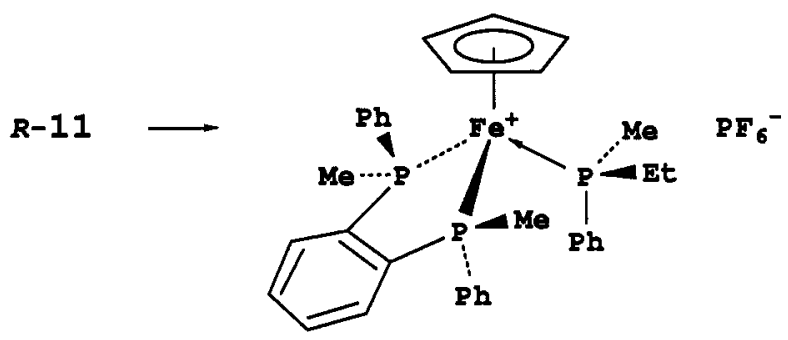

s-12

In recent work, we have discovered that 1,2-phenylenebis (phosphine) (ref. 13) can be peralkylated in the complex $\left[\mathrm{Cu}\left\{1,2-\mathrm{C}_{6} \mathrm{H}_{4}\left(\mathrm{PH}_{2}\right)_{2}\right\}\right] \mathrm{CF}_{3} \mathrm{SO}_{3}, 13$. Thus, treatment of 26 with $\mathrm{KOBu}-1$ ( 8 equiv.) and iodomethane ( 8 equiv.) at $20^{\circ} \mathrm{C}$ affords $1,2-$

phenylenebis (dimethylphosphine) in $80 \%$ yield after displacement from the metal. With dibromo-1,3-propane as alkylating agent, the tetrahedral cage 14 can be isolated in ca $50 \%$ yield. A full structural investigation of 14 and some of its complexes is in progress.

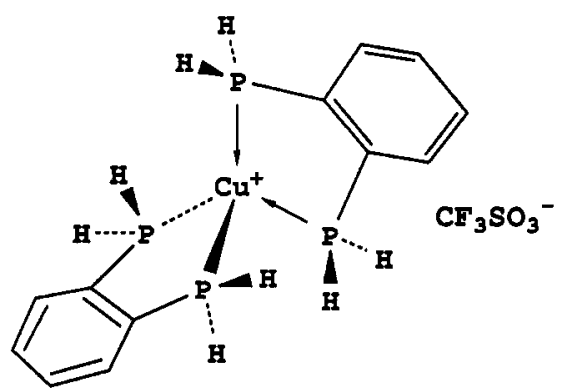

13

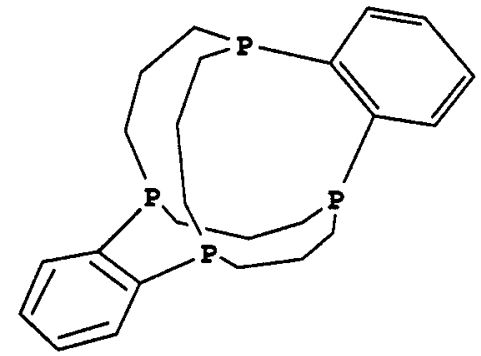

14 


\section{BIOLOGICAL SYNTHESIS OF CHIRAL TERTIARY ARSINES}

The mould Scopulariopsis brevicaulis in bread cultures reductively methylates prochiral dialkylarsinic acids under aerobic conditions to produce chiral tertiary arsines in considerable yields (ref. 14). We have shown that the reduction is highly stereoselective by trapping the tertiary arsines in solutions of optically active palladium complexes and analysing the products by high resolution ${ }^{1} \mathrm{H}$ NMR spectroscopy. Thus, when growing on bread treated with ethyl-n-propylarsinic acid, 15, the mould produces enantiomerically enriched ( \pm )-ethylmethyl-n-propylarsine, 16, of $80 \%$ optical purity. The progress of the reaction, as well as the stereoselectivity of the reaction, was monitored by the appearance of the diastereomers of the arsine with $R-6$. Present work is concerned with an investigation of the scope of this reaction with related substrates, as well as with the establishment of the identities of the major enantiomers of the products by X-ray analyses of crystalline palladium diastereomers. We are optimistic that the mould will produce optically active tertiary arsines, chiral at arsenic, with total stereoselectivity with arsinic acids containing bulkier alkyl substituents.<smiles>C[As](=O)(O)=[Pt]</smiles>

15

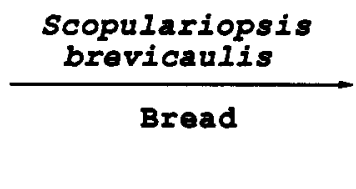

Bread

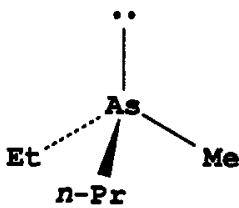

16

\section{REFERENCES}

1. J.D. Morrison (Ed.), Asymmerric Synthesis, Vols. 1-5, Academic Press, New York, 19831985.

2. K. Mislow, Trans. N.Y. Acad. Sci. 35, 227-242 (1973).

3. See, for example: D.J. Cram and J.M. Cram, Acc. Chem. Res. 8-14 (1978). J.-M. Lehn, Science (Washington. D.C.) 227, 849-856 (1985).

4. G.A. Melson, Coordination Chemistry of Macrocyclic Compounds, Plenum Press, New York, 1979.

5. T. Kauffman and J. Ennen, Chem. Ber. 118, 2692-2702, 2703-2713, 2714-2721 (1985).

6. P.G. Kerr, P.-H. Leung, and S.B. Wild, J. Am. Chem. Soc. 109, 4321-4328 (1987).

7. J.W.L. Martin, F.S. Stephens, K.D.V. Weerasuria, and S.B. Wild, J. Am. Chem. Soc. $110,4346-4356$ (1988).

8. G. Salem and S.B. Wild, J. Organomer. Chem. in press.

9. E.P. Kyba, C.N. Clubb, S.B. Larson, V.J. Schueler, and R.E. Davis, J. Am. Chem. Soc 107, 2141-2148 (1985) and references cited therein.

10. G.T. Crisp, G. Salem, F.S. Stephens, and S.B. Wild, J. Chem. Soc., Chem. Commun. 600602 (1987).

11. G. Salem and S.B. Wild, J. Chem. Soc., Chem. Commun. 1378 (1987).

12. G.T. Crisp, G. Salem, F.S. Stephens, and S.B. Wild, Organometallics in press.

13. E.P. Kyba, S.T. Liu, and R.L. Harris, Organomerallics 2, 1877-1879 (1983).

14. F. Challenger and A.A. Rawlings, J. Chen. Soc. 264-267 (1936). For a review on arsenic speciation in the environment, see V.R. Cullen and K.J. Reimer, Chem. Rev. in press. 